\title{
NUEVO OPERADOR DE AGREGACIÓN PARA GRUPOS DE PROCESOS
}

\author{
Federico Agostini ${ }^{1}$, David Luis La Red Martínez ${ }^{1}$ y Jorge Tomás Fornerón Martínez ${ }^{2}$ \\ ${ }^{1}$ Facultad de Cs. Ex. y Naturales y Agrimensura. Universidad Nacional del Nordeste. Corrientes, Argentina \\ ${ }^{2}$ Facultad de Ciencias Aplicadas. Universidad Nacional de Pilar. Pilar, Paraguay
}

\begin{abstract}
RESUMEN
Este documento describe cómo se pueden utilizar nuevos modelos de toma de decisiones en grupos de procesos distribuidos que requieren acceso a recursos compartidos en la modalidad de exclusión mutua. Los modelos de decisión actualmente disponibles y de aplicación general en los sistemas distribuidos se basan en algoritmos de intercambio de permisos que intentan alcanzar un acuerdo de todos los procesos que intervienen para realizar determinadas acciones. Una propuesta interesante es la creación de un operador de agregación que controle la asignación de recursos a grupos de procesos y un modelo de decisión que permita adaptarse a diferentes requerimientos, que los procesos accedan a recursos compartidos en la modalidad de exclusión mutua, que requiera o no sincronización, y que cumpla con ciertos requisitos para lograr el acceso a los recursos, y en base a esa información el modelo pueda elegir el operador de agregación correspondiente a cada caso. En cada nodo se define una interfaz entre las aplicaciones y el sistema operativo, que a través de un Runtime (software en tiempo de ejecución complementario al sistema operativo) incluido en dicha interfaz, gestiona los procesos y recursos compartidos y define el escenario correspondiente. Además, el Runtime interactúa entre sí para intercambiar información y hay un coordinador global de Runtime en uno de los nodos que evalúa y ejecuta el modelo de decisión y el operador de agregación correspondiente. El escenario propuesto en este documento considera la gestión de los recursos y procesos de los sistemas distribuidos, aplicados al entorno de las comunicaciones de datos, pero no se limitan a un protocolo de comunicaciones específico, es un esquema genérico.
\end{abstract}

\section{PALABRAS CLAVE}

Operadores de Agregación, Comunicación entre Grupos de Procesos, Exclusión Mutua, Sistemas Operativos

\section{INTRODUCCION}

La proliferación de sistemas informáticos, muchos de ellos distribuidos en diferentes nodos con múltiples procesos que cooperan para el logro de una determinada función, requiere modelos de decisión que permitan a grupos de procesos utilizar recursos compartidos a los que sólo se puede acceder en la modalidad de exclusión mutua. Las soluciones tradicionales para este problema se encuentran en (Tanenbaum, 1996 y 2009). Ambos documentos describen los principales algoritmos de sincronización en sistemas distribuidos. Los autores de (Ricart y Agrawala, 1981), (Cao y Singhal, 2001) y (Lodha y Kshemkalyani, 2000) presentan algoritmos para gestionar la exclusión mutua en redes informáticas. En (Stallings, 2005) se detallan los principales algoritmos para la gestión de procesos distribuidos, estados globales distribuidos y exclusión mutua distribuida. Asimismo, se han propuesto soluciones (que pueden considerarse tradicionales) para diferentes tipos de sistemas distribuidos en (Andrews, 2000), (Guerraoui y Rodrigues, 2006), (Lynch, 1996), (Tel, 2000) y (Attiya y Welch, 2004). En (Saxena y Rai, 2003) y (Velazquez, 1993) se han presentado otros trabajos centrados en garantizar la exclusión mutua. En este trabajo, se presentará un nuevo operador de agregación específicamente para resolver el problema mencionado anteriormente. Esto cae dentro de la categoría de operadores de OWA (Promedio Ponderado Ordenado). El uso de operadores de agregación en modelos de decisión ha sido ampliamente estudiado. Por ejemplo, (Greco et al, 2002), desarrolla metodologías que resuelven problemas en presencia de múltiples atributos y criterios y en (Chao et al, 2016) se estudia colectivamente la forma de obtener un vector prioritario, que se crea a partir de diferentes formatos de expresión de las preferencias de los tomadores de decisiones. Además, en (La Red Martínez y Acosta, 2015) se presenta las principales propiedades matemáticas y medidas de comportamiento relacionadas con los operadores de agregación. En (La Red Martínez y Pinto, 2015), (Yager, 1988 y 1993) se presenta una revisión de los 
operadores de agregación, especialmente los de la familia OWA. Los operadores OWA aplicados a la toma de decisiones multicriterio se presentan y analizan en (Yager y Kacprzyk, 1997), y (Yager y Pasi, 2002) analiza los operadores OWA y sus aplicaciones en el proceso de toma de decisiones.

Se presenta una variante de un método innovador para la gestión de recursos compartidos en sistemas distribuidos, basado en (La Red Martínez, 2017) y (La Red Martínez et al, 2018a y 2018b), donde se desarrollan operadores de agregación para asignar recursos en sistemas distribuidos, y en (Agostini et al, 2018 y 2019) donde se establecen modelos de consenso y se desarrollan operadores de agregación para favorecer el acceso secuencial de procesos o grupo de procesos a todos los recursos solicitados en las diferentes etapas de asignación de recursos a procesos. En este caso, se agrega un nuevo modelo, sin requerimientos de consenso (no se requiere consenso para asignar consecutivamente los recursos solicitados por un proceso, es decir, una vez que la secuencia de asignación de recursos al grupo ha comenzado, puede ser interrumpida para asignar recursos a otro proceso), con el objetivo de favorecer el acceso secuencial de los grupos de procesos a todos los recursos solicitados. Las premisas, estructuras de datos y operadores mencionados en (La Red Martínez, 2017), (La Red Martínez et al, 2018a y 2018b), (Agostini et al, 2018 y 2019), y (Agostini y La Red Martínez, 2019) se utilizan como punto de partida para crear un nuevo operador y establecer el modelo de decisión para el escenario mencionado.

\section{ESTRUCTURA DE DATOS}

\subsection{Escenario Propuesto}

Este escenario se basa en las publicaciones de los escenarios mencionados anteriormente y es también un caso particular de (Agostini y La Red Martínez, 2019), pero en ausencia de consenso, las asignaciones se hacen en rondas, en lugar de tomar las prioridades globales de todas las rondas como en los escenarios mencionados, ver Figura 1.
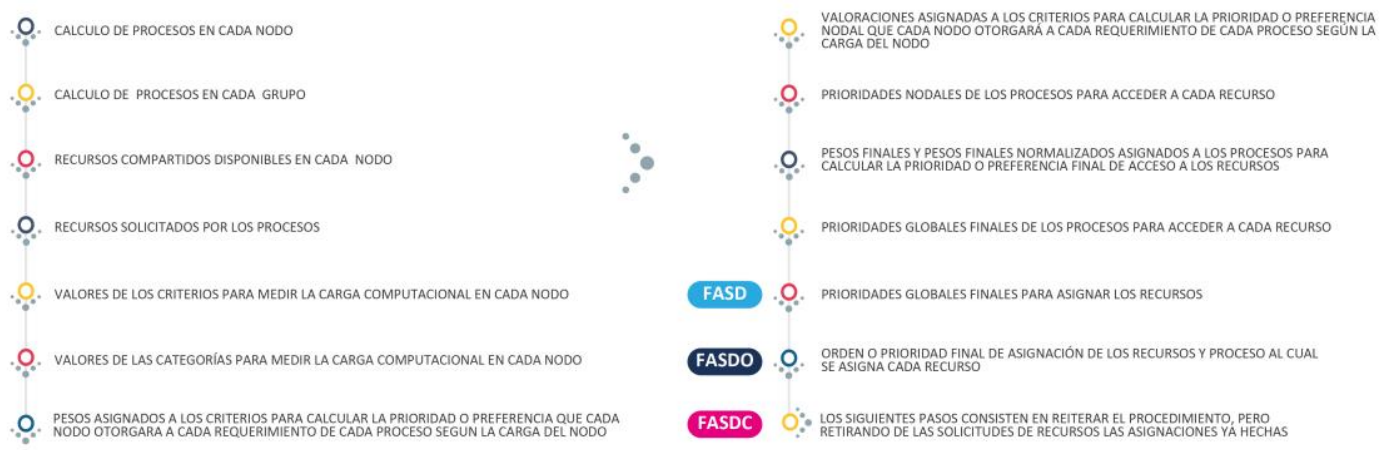

Figura 1. Pasos para calcular la Función de Asignación para Sistemas Distribuidos (FASD)

Los procesos no requieren sincronización (estar activos en sus respectivos procesadores en el mismo lapso de tiempo) ni estrictos requisitos de consenso para lograr el acceso.

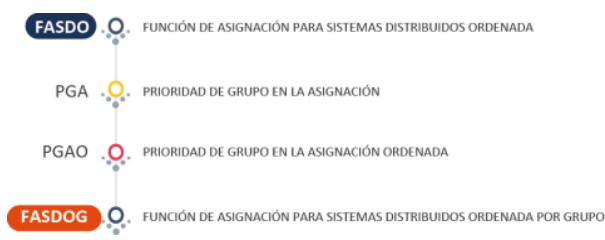

Figura 2. Pasos para obtener desde la $F A S D O$ a la $F A S D O G$

En la Figura 2 se muestra una lista de los pasos necesarios para obtener las prioridades globales definitivas para asignar los recursos (Función de Asignación para Sistemas Distribuidos Ordenada- FASDO) hasta obtener el orden o prioridad de asignación de los recursos y el proceso al que se asigna cada recurso ordenada por grupo (Función de Asignación para Sistemas Distribuidos Ordenada por Grupo - FASDOG). 
Una vez calculada la $F A S D O$, se establecerá la prioridad grupal y el orden en el que se asignarán los recursos a procesos en los grupos. Para ello se tendrá en cuenta todos los procesos y recursos vinculados en cada instancia. La cardinalidad de grupo se medirá por la cantidad de procesos que hay en cada grupo, en cada instancia.

\subsection{Prioridad del Grupo en la Asignación}

La sumatoria de todas las prioridades finales $(F A S D O)$ de cada relación proceso y recurso por grupo, dividido por la cardinalidad (números de asignaciones de cada grupo, en cada instancia) del grupo, indicará la prioridad que tendrá dicho grupo para ser asignado, en relación a los demás grupos que también tendrán que ser asignados. Los procesos independientes, es decir, que no formen grupos, serán agrupados y considerados como el último grupo, su cardinalidad será el número de asignaciones que lo conformen y su prioridad cero.

Esto constituye lo que se denominará Prioridad de Grupo en la Asignación ( $P G A)$ :

$P G A_{i}$ con $i=1, \ldots, g=\left(\sum F A S D O_{j}\right) /$ (cardinalidad del grupo $i$ en la asignación)

$g=$ total de grupos en la asignación; $j=\mathrm{n}^{\circ}$ de asignaciones del grupo $i$ en la ronda (se tiene en cuenta una asignación para cada uno de los recursos disponibles).

Del vector $P G A$ se debe ordenar sus elementos de mayor a menor para obtener el orden prioritario del grupo en la ronda de asignación.

$P G A O_{i}=$ Prioridad del Grupo en la Asignación Ordenada $\mathrm{g}=$ cardinalidad de $P G A$ (total de grupos en la asignación)

$P G A O_{i}=\operatorname{Max}\left(P G A_{i}\right.$ no ordenado $) \quad i=1, \ldots, g$

No ordenado $=P G A_{i} \notin P G A O$

Orden de prioridad global de los grupos en la asignación, ver Figura 3:
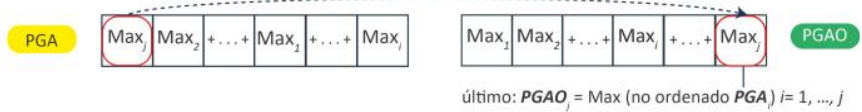

Figura 3. Ejemplo para calcular la $P G A O$ de cada grupo de procesos

\subsection{Función de Asignación para Sistemas Distribuidos Ordenada por Grupo (FASDOG)}

La FASDOG establecerá el orden de asignación por prioridades de grupo de los procesos para acceder a sus recursos y se establecerá el orden en el que se asignará cada uno, ver Figura 4.
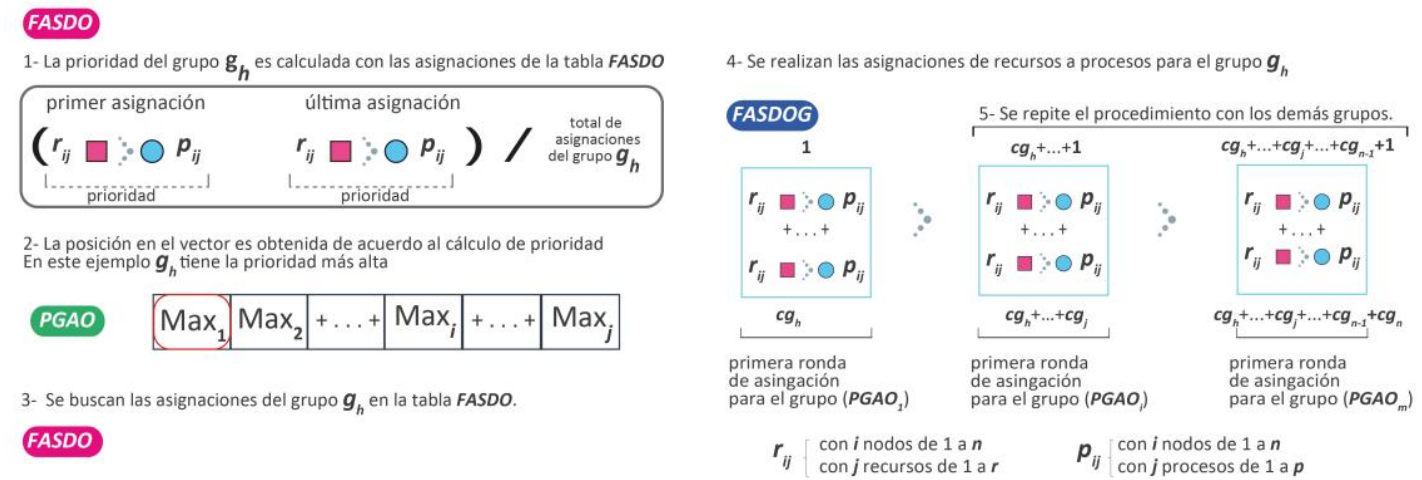

Figura 4. Función de asignación de recursos por grupo

Para ello, se tendrá en cuenta la tabla $F A S D O$ y la $P G A O$.

Se calcularán las cardinalidades (número de asignaciones de recursos a procesos) obtenidas de cada uno de los grupos del vector $P G A O$ en la tabla $F A S D O$, en la ronda.

$c g_{i}=$ cardinalidad $\operatorname{grupo}\left(P G A O_{i}\right)$ en $F A S D O$

Luego habrá que obtener cada una de las asignaciones de recursos a procesos en la tabla FASDO de cada 
uno de los grupos del vector PGAO. El total de elementos por cada grupo, estará determinado por la cardinalidad calculada en el paso anterior.

El siguiente paso es repetir el procedimiento, pero eliminando las solicitudes de asignaciones ya realizadas en la primera ronda; debe tenerse en cuenta que los recursos asignados estarán disponibles una vez liberados por los procesos y, por lo tanto, pueden asignarse a otros grupos de procesos en la siguiente ronda.

\subsection{Ejemplo}

El escenario que se presenta a continuación, parte de la asignación ordenada de la primera iteración correspondiente a la tabla FASDO obtenida en (Agostini et al, 2018), al cual se le han agregado dos procesos independientes.

La Tabla 1 muestra el orden de las asignaciones de recursos para cada proceso, ordenado por proceso con mayor prioridad en la asignación.

Tabla 1. Orden de asignación en la FASDO

\begin{tabular}{lllll}
\hline Prioridad & Recursos & Procesos & Rondas & Grupo \\
\hline 0.4730645 & $r_{12}$ & $p_{37}$ & 1 & $g_{4}$ \\
0.4679839 & $r_{33}$ & $p_{23}$ & 1 & $g_{1}$ \\
0.3704839 & $r_{31}$ & $p_{34}$ & 1 & $g_{1}$ \\
0.3642230 & $r_{14}$ & $p_{12}$ & 1 & \\
0.3512097 & $r_{11}$ & $p_{37}$ & 1 & $g_{4}$ \\
0.3440323 & $r_{22}$ & $p_{34}$ & 1 & $g_{1}$ \\
0.3300000 & $r_{21}$ & $p_{37}$ & 1 & $g_{4}$ \\
0.3291231 & $r_{34}$ & $p_{38}$ & 1 & \\
0.3286290 & $r_{13}$ & $p_{13}$ & 1 & $g_{1}$ \\
0.3032258 & $r_{32}$ & $p_{34}$ & 1 & $g_{1}$ \\
0.2491936 & $r_{23}$ & $p_{11}$ & 1 & $g_{4}$ \\
0.1895161 & $r_{24}$ & $p_{34}$ & 1 & $g_{1}$ \\
\hline \multicolumn{5}{c}{ Fuente: Elaboración propia. }
\end{tabular}

Una vez que se haya completado la tabla de la $F A S D O$, con la información de grupo de cada proceso, se calcularán las Prioridad del Grupo en la Asignación $(P G A)$ :

$P G A_{l} \Rightarrow>(0.4679839+0.3704839+0.3440323+0.3286290+0.3032258+0.1895161) / 6=0.33397850$

$P G A_{2} \Rightarrow(0.4730645+0.3512097+0.3300000+0.2491936) / 4=0.35086695$

$P G A_{3}=>0$

En este ejemplo, se pueden observar procesos que pertenecen a dos grupos $g_{1}$ y $g_{4}$, y dos procesos independientes que no pertenecen a grupos. Estos últimos son agrupados $\left(g_{0}\right)$, su cardinalidad es dos y su prioridad es 0 .

Al calcular el $P G A$ para todos los grupos, se obtendrá un vector como se muestra en la Tabla 2. Del vector $P G A$ se debe ordenar sus elementos de mayor a menor para obtener el orden prioritario de asignación a grupos, como se puede observar en la

Tabla 3 .

La FASDOG establecerá el orden de asignación por prioridades de grupo de los procesos para acceder a sus recursos y se establecerá el orden en el que se asignará cada uno. Para ello, se tendrá en cuenta la tabla FASDO y la $P G A O$.

Tabla 2. PGA (prioridad de grupo en la asignación)

\begin{tabular}{ll}
\hline Prioridad Global Final & Grupo \\
\hline 0.33397850 & $g_{1}$ \\
0.35086695 & $g_{4}$ \\
0 & $g_{0}$ \\
\hline & Fuente: Elaboración propia.
\end{tabular}

Tabla 3. PGA (prioridad de grupo en la asignación)

\begin{tabular}{ll}
\hline Prioridad Global Final Ordenada & Grupo \\
\hline 0.35086695 & $g_{4}$ \\
0.33397850 & $g_{1}$ \\
0 & $g_{0}$ \\
\hline
\end{tabular}

Fuente: Elaboración propia. 
Se calcularán las cardinalidades (número de asignaciones de recursos a procesos) obtenidas de cada uno de los grupos del vector $P G A O$ en la tabla $F A S D O$, de la primera asignación.

Luego habrá que obtener cada una de las asignaciones de recursos a procesos en la tabla FASDO de cada uno de los grupos del vector PGAO. El total de elementos por cada grupo, estará determinado por la cardinalidad calculada en el paso anterior.

Cálculo de las $F A S D O G$ para el primer grupo:

$F A S D O G_{1}=$ primer elemento de la $F A S D O$ para el grupo $\left(P G A O_{1}\right)$

...

$F A S D O G_{4}=$ último elemento de la $F A S D C O$ para el grupo $\left(P G A O_{1}\right)$

Cálculo de las FASDOG para el segundo grupo

$F_{A S D O G_{4+1}}=$ primer elemento de la $F A S D O$ para el grupo $\left(P G A O_{2}\right)$

...

$F A S D O G_{4+6}=$ último elemento de la $F A S D C O$ para el grupo $\left(P G A O_{2}\right)$

Cálculo de las $F A S D O G$ para el tercer grupo

$F A S D O G_{4+6+1}=$ primer elemento de la $F A S D O$ para el grupo $\left(P G A O_{3}\right)$

$F A S D O G_{4+6+2}=$ último elemento de la $F A S D C O$ para el grupo $\left(P G A O_{3}\right)$

La Tabla 4 muestra el orden final de asignación para la primera iteración de la FASDOG.

Tabla 4. Orden de asignación de grupo en la FASDOG (primera iteración)

\begin{tabular}{lllll}
\hline Prioridad & Recursos & Procesos & Rondas & Grupo \\
\hline 0.4730645 & $r_{12}$ & $p_{37}$ & 1 & $g_{4}$ \\
0.3512097 & $r_{11}$ & $p_{37}$ & 1 & $g_{4}$ \\
0.3300000 & $r_{21}$ & $p_{37}$ & 1 & $g_{4}$ \\
0.2491936 & $r_{23}$ & $p_{11}$ & 1 & $g_{4}$ \\
0.4679839 & $r_{33}$ & $p_{23}$ & 1 & $g_{1}$ \\
0.3704839 & $r_{31}$ & $p_{34}$ & 1 & $g_{1}$ \\
0.3440323 & $r_{22}$ & $p_{34}$ & 1 & $g_{1}$ \\
0.3286290 & $r_{13}$ & $p_{13}$ & 1 & $g_{1}$ \\
0.3032258 & $r_{32}$ & $p_{34}$ & 1 & $g_{1}$ \\
0.1895161 & $r_{24}$ & $p_{34}$ & 1 & $g_{1}$ \\
0.3642230 & $r_{14}$ & $p_{12}$ & 1 & \\
0.3291231 & $r_{34}$ & $p_{38}$ & 1 & \\
\hline \multicolumn{5}{c}{ Fuente: Elaboración propia. }
\end{tabular}

El siguiente paso es repetir el procedimiento, pero eliminando las solicitudes de asignaciones ya realizadas en la primera ronda; debe tenerse en cuenta que los recursos asignados estarán disponibles una vez liberados por los procesos y, por lo tanto, pueden asignarse a otros grupos de procesos en la siguiente ronda.

\section{DESCRIPCIÓN DEL OPERADOR DE AGREGACIÓN}

En (La Red Martínez, 2017), (La Red Martínez et al, 2018a y 2018b), (Agostini et al, 2018 y 2019), y (Agostini y La Red Martínez, 2019), las características de los operadores de agregación descritos permiten considerar que el método propuesto pertenece a la familia de operadores de agregación Neat-OWA. La propuesta desarrollada en este trabajo consiste en generar un modelo de decisión y su correspondiente operador de agregación para la gestión de grupos de procesos que comparten recursos, realizando modificaciones de los operadores de la familia OWA mencionados.

\section{MODELO DE DECISIÓN}

El objetivo principal del modelo propuesto es considerar el entorno de ejecución distribuida de procesos, que podrán estar agrupados, con distintas exigencias de consenso respecto del acceso a recursos compartidos, para seleccionar el método de agregación más adecuado a cada escenario posible, para generar la secuencia de 
asignación de los recursos a los procesos que los solicitan, respetando la exclusión mutua en el acceso a dichos recursos.

En la Figura 5 se puede observar que el modelo propuesto comienza con el cálculo de la FASD (La Red Martínez, 2017) y (La Red Martínez et al, 2018a y 2018b), luego puede aplicarse según el escenario, el operador propuesto en (Agostini et al, 2018 y 2019), sin formar grupos pero con consenso para lograr el acceso a los recursos, el operador presentado en (Agostini y La Red Martínez, 2019) que considera grupos de procesos con requerimientos de consenso, y por último lo que se muestra como escenario 4, el método propuesto en este trabajo, grupos de procesos pero sin consenso para lograr el acceso a los recursos.

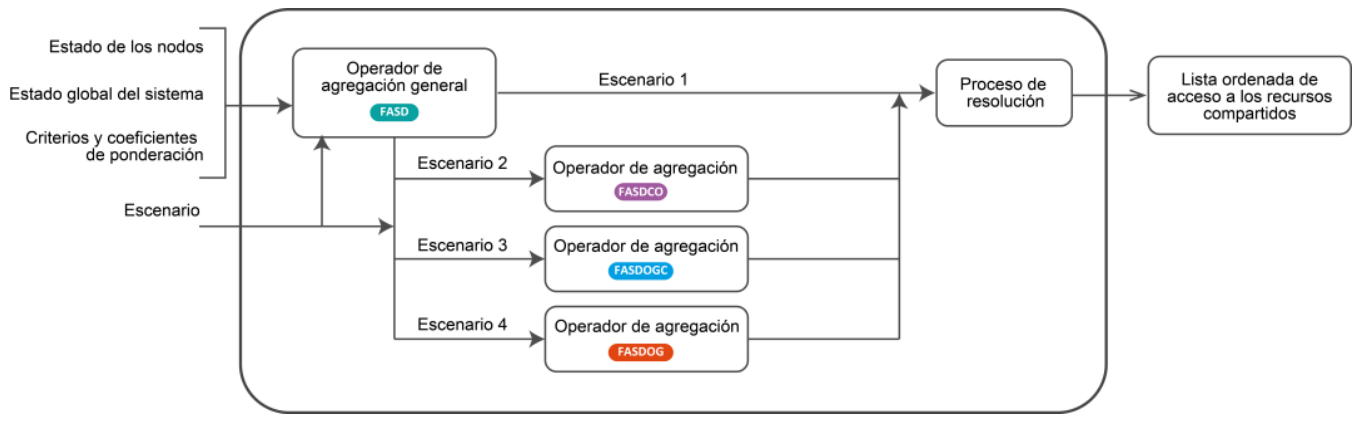

Figura 5. Modelo de decisión propuesto

En el modelo propuesto, en cada nodo se define una interfaz entre las aplicaciones y el sistema operativo, que a través de un Runtime (software en tiempo de ejecución complementario del sistema operativo) incluído en esa interfaz, gestiona los procesos y recursos compartidos y define el escenario correspondiente. Además los Runtime interactúan entre sí para intercambiar información y existe un Runtime coordinador global en uno de los nodos que evalúa y ejecuta el modelo de decisión y el operador de agregación correspondiente, ver Figura 6.

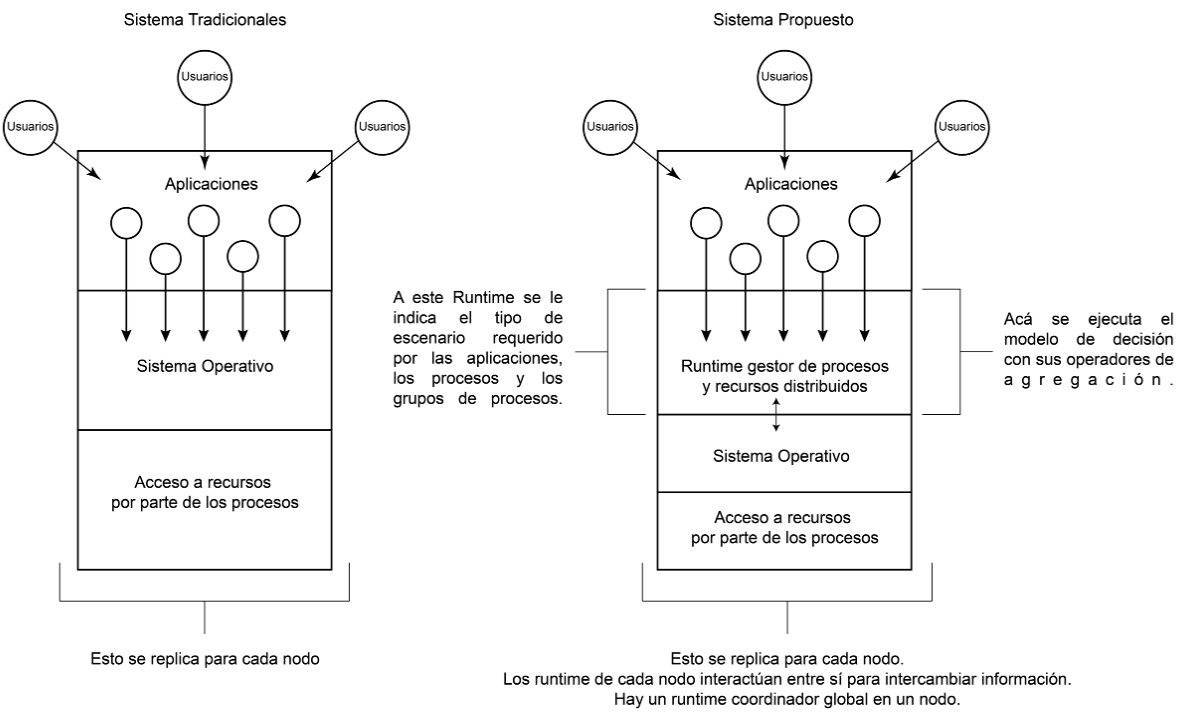

Figura 6. Comparación del Modelo propuesto y los modelos tradicionales

Comparación de las diferencias más significativas del método propuesto con respecto a los métodos tradicionales, se considera el algoritmo centralizado, el Algoritmo Distribuido de Lamport, Ricart y Agrawala y el Algoritmo de Token Ring (Colouris et al, 1988) y (Tanenbaum y Van Steen, 2001). El algoritmo propuesto establece el orden inicial de asignación por prioridades, mientras que los demás lo hacen teniendo en cuenta la timestamp. Los requisitos de acceso a recursos en el algoritmo propuesto se establecen calculando prioridades, mientras que en el algoritmo centralizado es por mensajes al coordinador, el Algoritmo Distribuido de Lamport, Ricart y Agrawala a través de mensajes a procesos $n-1$ y el Algoritmo Token Ring a través de mensajes a sus vecinos. El método de asignación en el algoritmo propuesto contempla la mayor prioridad, mientras que en los 
demás se tiene en cuenta la timestamp más antiguo. Sólo el método propuesto tiene en cuenta los grupos de procesos, mientras que los demás sólo tienen en cuenta procesos independientes. La predisposición (prioridad nodal), carga de nodos, estado nodal (nodos, procesos, grupos, recursos) y estado global del sistema, sólo se tiene en cuenta en el algoritmo propuesto.

\section{CONCLUSION}

\subsection{Evaluación}

El modelo propuesto logra establecer un orden que permite a los grupos de procesos acceder a todos sus recursos de manera secuencial y que éstos no pueden ser removidos hasta que el grupo de procesos que los mantiene los libere. El orden de asignación será determinado por la prioridad promedio general de cada grupo en cada asignación. El sistema distribuido regula y actualiza constantemente el estado local de cada nodo, las decisiones de acceso a los recursos modifican estos estados por lo que debe ser reajustado repetidamente, garantizando la exclusión mutua y reordenando nuevas prioridades. El método debe repetirse siempre que haya grupos de procesos que requieran recursos compartidos.

Este trabajo considera que el grupo de procesos al cual se le otorgan los recursos, se determina con el promedio global de prioridades en todas las asignaciones en la ronda $P G A O$, pero el orden en el que se deben realizar esas asignaciones, respeta el de la tabla $F A S D O$, para cada grupo de procesos. El modelo propuesto incluye, como caso particular, un método que consiste en considerar la prioridad de los grupos de procesos en cada ronda de asignación, en lugar de procesos independientes.

La estructura de datos mencionada anteriormente y el método de agregación utilizado no están totalmente cubiertos por los métodos tradicionales, por ejemplo, no contemplan la predisposición (prioridad nodal), carga de nodos, estado nodal (nodos, procesos, grupos, recursos...) ni el estado global del sistema, para el cálculo de prioridades en la asignación de recursos a los procesos.

\subsection{Futuras Líneas de Investigación}

Como trabajo futuro, se prevé desarrollar variantes del método propuesto considerando otros operadores de agregación (especialmente la familia OWA), utilizando etiquetas lingüísticas para establecer los criterios de evaluación de los estados nodales, la consideración de la migración controlada de procesos, con el objetivo de disminuir el tráfico de la red en sistemas distribuidos y la clasificación de grupos de procesadores para la agrupación de nodos según rangos que cumplan ciertas características (velocidad, tiempo de cálculo, procesamiento, etc.).

Se considera desarrollar modelos de decisión desde la óptica cognitiva para la toma de decisiones en grupos de procesos, contemplando los principios de la cibernética de segundo orden, en el contexto de sistemas complejos de auto-regulación, que trasciendan el enfoque tradicional de las ciencias de la computación considerando la posibilidad de imputación de datos faltantes. Se desarrollará un simulador en el que se considerarán los diferentes escenarios mencionados para estudiar el comportamiento de los métodos propuestos y de los clásicos para las mismas cargas de trabajo.

\section{AGRADECIMIENTO}

Este trabajo ha sido apoyado por el Proyecto: "Modelos de decisión y operadores de agregación para la gestión de procesos en sistemas distribuidos", código 16F001 de la Universidad Nacional del Nordeste (Argentina). 


\section{REFERENCIAS}

Agostini F. et al, 2018. Modeling of the Consensus in the Allocation of Resources in Distributed Systems. International Journal of Advanced Computer Science and Applications (IJACSA). Vol. 9, No. 12.

Agostini F. et al, 2019. Assignment of Resources in Distributed Systems with Strict Consensus Requirements, 10th International Multi-Conference on Complexity, Informatics and Cybernetics (IMCIC). Orlando, USA. http://dx.doi.org/10.14569/JJACSA.2018.091204.

Agostini F., La Red Martínez D. L., 2019. Allocation of shared resources strict consensus model for process groups. Asociación Ibérica de Sistemas y Tecnologías de Información. Coimbra, Portugal.

Andrews G., 2000. Foundation of Multithreaded, Parallel, and Distributed Programming. Reading, MA: Addison-Wesley, USA.

Attiya H., Welch J., 2004. Distributed Computing Fundamentals, Simulations, and Advanced Topics, John Wiley, 2nd ed.. New York, USA.

Cao G., Singhal M., 2001. A Delay-Optimal Quorum-Based Mutual Exclusion Algorithm for Distributed Systems. IEEE Transactions on Parallel and Distributed Systems. USA .Vol. 12, No. 12, pp 1256-1268.

Chao X. et al, 2016. An optimization model integrating different preference formats. 6th International Conference on Computers Communications and Control (ICCCC). pp $228-231$.

Colouris G. et al, 1988. Distributed Systems. Concepts and Design. Pearson.

Greco S. et al, 2002. Rough sets methodology for sorting problems in presence of multiple attributes and criteria. European Journal of Operational Research. Vol. 138, pp 247-259.

Guerraoui R., Rodrigues L., 2006. Introduction to Reliable Distributed Programming, Berlin, Springer-Verlag.

La Red Martínez D. L. et al, 2018a. Assignment of Resources in Distributed Systems. 9th International Multi-Conference on Complexity, Informatics and Cybernetics (IMCIC 2018). Orlando, USA.

La Red Martínez D. L. et al, 2018b. Assignment of Resources in Distributed Systems. Journal on Systemics, Cybernetics and Informatics: JSCI. Vol. 16, No. 5, pp 81-87.

La Red Martínez D. L., 2017. Aggregation Operator for Assignment of Resources in Distributed Systems. International Journal of Advanced Computer Science and Applications (IJACSA). The Science and Information (SAI) Organization, England. U.K. Vol. 8, No. 10, pp 406-419, ISSN N ${ }^{\circ} 2156-5570$.

La Red Martínez D. L., Acosta J. C., 2015. Aggregation Operators Review - Mathematical Properties and Behavioral Measures. International Journal of Intelligent Systems and Applications (IJISA). Hong Kong, Vol. 7, No. 10, pp 63-76.

La Red Martínez D. L., Pinto N., 2015. Brief Review of Aggregation Operators. Wulfenia Journal. Austria, Vol. 22, No. 4, pp 114-137.

Lodha S., Kshemkalyani A., 2000. A Fair Distributed Mutual Exclusion Algorithm. IEEE Trans. Parallel and Distributed Systems. USA. Vol. 11, No. 6, pp 537-549.

Lynch N., 1996. Distributed Algorithms. Morgan Kauffman, San Mateo, CA, USA.

Ricart G., Agrawala A. K., 1981. An Optimal Algorithm for Mutual Exclusion in Computer Networks. Commun. of the $A C M$, Vol. 24, pp 9-17.

Saxena P., Rai J., 2003. A Survey of Permission-based Distributed Mutual Exclusion Algorithms. Computer Standards and Interfaces. Vol. 25, No. 2, pp 159-181.

Stallings W., 2005. Sistemas Operativos, 5ta. Edición, Pearson Educación S.A.. España.

Tanenbaum A. S., 1996. Sistemas Operativos Distribuidos. Prentice - Hall Hispanoamericana S.A., México.

Tanenbaum A. S., 2009. Sistemas Operativos Modernos. 3ra. Edición. Pearson Educación S. A., México.

Tanenbaum A. S., Van Steen M., 2001. Distributed Systems: Principles and Paradigms. Pearson.

Tel G., 2000. Introduction to Distributed Algorithms, Cambridge University Press, 2nd ed. Cambridge, UK.

Velazquez M., 1993. A Survey of Distributed Mutual Exclusion Algorithms. Technical Report CS-93-116, University of Colorado at Boulder.

Yager R., 1988. On Ordered Weighted Averaging Aggregation Operators in Multi-Criteria Decision Making. IEEE Trans. On Systems, Man and Cybernetics. Vol. 18, No. 1, pp 183-190.

Yager R., 1993. Families of OWA Operators. Fuzzy Sets and Systems. Vol. 59, No. 2, pp 125-148.

Yager R., Kacprzyk J., 1997. The Ordered Weighted Averaging Operators. Theory and Applications, Kluwer Academic Publishers. USA.

Yager R., Pasi G., 2002. Modelling Majority Opinion in Multi-Agent Decision Making. International Conference on Information Processing and Management of Uncertainty in Knowledge-Based Systems. 